\title{
A SOLUTION OF A SIMPLE FUNCTIONAL EQUATION AS A BASIS FOR READILY OBTAINING CERTAIN FUNDAMENTAL FORMULAS IN THE THEORY OF ELLIPTIC FUNCTIONS*
}

BY G. W. STARCHER

The purpose of the present note is to show, by considering certain solutions of simple functional equations, how one may obtain expressions for each of the four theta functions, both as infinite products and as Fourier series, and thus have a basis for the theory of elliptic functions. The principal novelty of the note lies in the fact that the two fundamental expressions for the theta functions are obtained at once from considering simple functional equations; moreover, an important relation between theta functions of zero argument is obtained with less effort than is usually given to it. Throughout this discussion we assume $q$ to be such that $|q|<1$.

The functional equation

$$
f(x)=(1+q x) f(q x)
$$

has a unique solution analytic in the neighborhood of the point zero, having there the value 1 ; this solution is expressible in each of the two forms

(2) $\prod_{v=1}^{\infty}\left(1+q^{v} x\right)=1+\sum_{v=1}^{\infty} \frac{q^{(1 / 2) v(v+1)}}{(1-q)\left(1-q^{2}\right) \cdots\left(1-q^{v}\right)} x^{v}$.

From (1), we see that $g(x)=1 / f(-x)$ satisfies the equation

$$
g(x)=\frac{1}{1-q x} g(q x) .
$$

This equation has a unique solution analytic in the neighborhood of the point zero, having there the value 1 , which is expressible as an infinite product by comparison with the expression for $f(x)$ as an infinite product. If such a solution of (3) may be written in the form

\footnotetext{
* Presented to the Society, April 18, 1930.
} 


$$
g(x)=1+\sum_{v=1}^{\infty} \frac{c_{v} x^{v}}{(1-q x)\left(1-q^{2} x\right) \cdots\left(1-q^{v} x\right)},
$$

then we have

$$
\begin{aligned}
& \frac{1}{1-q x} g(q x)=\frac{1}{1-q x} \\
& \quad+\sum_{v=1}^{\infty} \frac{c_{v} q^{v} x^{v}}{(1-q x)\left(1-q^{2} x\right) \cdots\left(1-q^{v} x\right)} \cdot \frac{1}{\left(1-q^{v+1} x\right)} \\
& \quad 1+\frac{q x}{1-q x}+\sum_{v=1}^{\infty} \frac{c_{v} q^{v} x^{v}}{(1-q x)\left(1-q^{2} x\right) \cdots\left(1-q^{v} x\right)} \\
& \quad+\sum_{v=1}^{\infty} \frac{c_{v} q^{2 v+1} x^{v+1}}{(1-q x)\left(1-q^{2} x\right) \cdots\left(1-q^{v+1} x\right)} .
\end{aligned}
$$

Equating coefficients of like terms and computing the successive $c$ 's we have finally the solution

$$
g(x)=1+\sum_{v=1}^{\infty} \frac{q^{v}}{(1-q)\left(1-q^{2}\right) \cdots\left(1-q^{v}\right)} \frac{x^{v}}{(1-q x)\left(1-q^{2} x\right) \cdots\left(1-q^{v} x\right)} .
$$

For $x=1$, we have

$$
\frac{1}{\prod_{v=1}^{\infty}\left(1-q^{v}\right)}=1+\sum_{v=1}^{\infty} \frac{q^{v}}{(1-q)^{2}\left(1-q^{2}\right)^{2} \cdots\left(1-q^{v}\right)^{2}}
$$

Consider the functional equation

$$
f(x)=x f(q x) .
$$

If there exists a solution of this equation, not identically zero, which vanishes when $x=-1$, having no other zeros $x_{1}$ such that $|q|<\left|x_{1}\right| \leqq 1$, then all the zeros of such a solution are at the points $x=-q^{\nu}$, where $\nu=\cdots,-2,-1,0,1,2, \cdots$. We may construct a function having precisely these zeros, viz.,

$$
f_{1}(x)=\prod_{v=1}^{\infty}\left(1+q^{v-1} x\right)\left(1+q^{v} x^{-1}\right) .
$$

This infinite product obviously converges and defines a singlevalued analytic function for every $x$ except $x=0$ and $x=\infty$. Substituting $f_{1}(x)$ for $f(x)$ in (5) we see that $f_{1}(x)$ satisfies (5). 
Any solution of (5) which is single-valued and analytic throughout the plane except at $x=0$ and $x=\infty$, may be represented by a Laurent series which may be written,

$$
f(x)=\sum_{-\infty}^{\infty} c_{v} x^{v}
$$

Substituting from (7) into (5) we have

$$
\sum_{-\infty}^{\infty} c_{v} x^{v}=\sum_{-\infty}^{\infty} c_{v} q^{v} x^{v+1}
$$

Computing the successive $c$ 's in terms of $c_{0}$ and substituting these values into (7) we have

$$
f(x)=c_{0} \sum_{-\infty}^{\infty} q^{(1 / 2) v(v-1)} x^{v} .
$$

This series converges and defines a function which is singlevalued and analytic throughout the plane except at $x=0$ and $x=\infty$. Hence a solution of (5) which is single-valued and analytic throughout the plane except at $x=0$ and $x=\infty$ is uniquely determined by (5) except for a multiplier $c_{0}$ which is independent of $x$. For some particular $c_{0}$ the series in (8) must define the same solution as (6). To find the required value of $c_{0}$, we observe that $c_{0}$ is the term in the series that is independent of $x$, and determine the term independent of $x$ in the expansion of the infinite product (6). From (2) when $x=q^{-1} x$ we have

$$
\prod_{v=1}^{\infty}\left(1+q^{v-1} x\right)=1+\sum_{v=1}^{\infty} \frac{q^{(1 / 2) v(v-1)}}{(1-q)\left(1-q^{2}\right) \cdots\left(1-q^{v}\right)} x^{v}
$$

and when $x=x^{-1}$

$$
\prod_{v=1}^{\infty}\left(1+q^{v} x^{-1}\right)=1+\sum_{v=1}^{\infty} \frac{q^{(1 / 2) v(v+1)}}{(1-q)\left(1-q^{2}\right) \cdots\left(1-q^{v}\right)} x^{-v} .
$$

In the product of the two expressions on the right the sum of the products of terms involving $x^{v}$ in the first by the terms involving $x^{-v}$ in the second, together with the product of the two constant terms, gives precisely the term independent of $x$ in the expansion of (6). This is clearly just the second mem- 
ber of the identity (4). Writing the first member of (4) for $c_{0}$ in (8) and equating to the product in (6) we obtain

(9) $\prod_{v=1}^{\infty}\left(1+q^{v-1} x\right)\left(1+q^{v} x^{-1}\right)\left(1-q^{v}\right)=\sum_{-\infty}^{\infty} q^{(1 / 2) v(v-1)} x^{v}$.

Replacing $x$ by $-x$ we also have

(10) $\prod_{v=1}^{\infty}\left(1-q^{v-1} x\right)\left(1-q^{v} x^{-1}\right)\left(1-q^{v}\right)=\sum_{-\infty}^{\infty}(-1)^{v} q^{(1 / 2) v(v-1)} x^{v}$.

Replacing $q$ by $q^{2}$ and $x$ by $q e^{2 i z}$ and using the customary notation $\vartheta_{3}(z), \vartheta_{1}(z)$ for the theta functions, we have from (9) and (10)

$$
\begin{aligned}
& \prod_{v=1}^{\infty}\left(1+q^{2 v-1} e^{2 i z}\right)\left(1+q^{2 v-1} e^{-2 i z}\right)\left(1-q^{2 v}\right) \\
= & \sum_{-\infty}^{\infty} q^{v^{2}} e^{2 v i z}=\imath^{9}{ }_{3}(z), \\
& \prod_{v=1}^{\infty}\left(1-q^{2 v-1} e^{2 i z}\right)\left(1-q^{2 v-1} e^{-2 i z}\right)\left(1-q^{2 v}\right) \\
= & \sum_{-\infty}^{\infty}(-1)^{v} q^{v^{2}} e^{2 v i z}={ }_{4}(z) .
\end{aligned}
$$

Again replacing $q$ by $q^{2}$ and $x$ by $q^{2} e^{2 i z}$, we have from (9) after multiplying by $q^{1 / 4} e^{i z}$

$$
\begin{gathered}
q^{1 / 4} e^{i z} \prod_{v=1}^{\infty}\left(1+q^{2 v} e^{2 i z}\right)\left(1+q^{2 v-2} e^{-2 i z}\right)\left(1-q^{2 v}\right) \\
=\sum_{-\infty}^{\infty} q^{(v+1 / 2)^{2}} e^{(2 v+1) i z}=\vartheta_{2}(z) .
\end{gathered}
$$

Similarly from (10) after multiplying by $-i q^{1 / 4} e^{i z}$

$$
\begin{gathered}
-i q^{1 / 4} e^{i z} \prod_{v=1}^{\infty}\left(1-q^{2 v} e^{2 i z}\right)\left(1-q^{2 v-2} e^{-2 i z}\right)\left(1-q^{2 v}\right) \\
=\sum_{-\infty}^{\infty}(-1)^{v} q^{(v+1 / 2)^{2}} e^{(2 v+1) i z}=\vartheta_{1}(z)
\end{gathered}
$$


Thus we have each of the four theta functions expressed as an infinite product and as an infinite series, the latter being equivalent to the Fourier series for the theta functions. From these it is easy to establish the quasi-periodic character of the theta functions, together with other of their fundamental properties. By considering suitable quotients of the theta functions we obtain elliptic functions, and hence much of the theory of elliptic functions may be obtained readily.

We may easily obtain a fundamental relation between the theta functions of zero argument as follows.

From (14) we may write

$$
\begin{aligned}
\vartheta_{1}(z) & =-i q^{1 / 4} e^{i z}\left(1-e^{-2 i z}\right) \prod_{v=1}^{\infty}\left(1-q^{2 v} e^{2 i z}\right)\left(1-q^{2 v} e^{-2 i z}\right)\left(1-q^{2 v}\right) \\
& =2 q^{1 / 4} \sin z \prod_{v=1}^{\infty}\left(1-q^{2 v} e^{2 i z}\right)\left(1-q^{2 v} e^{-2 i z}\right)\left(1-q^{2 v}\right) .
\end{aligned}
$$

Differentiating with respect to $z$, then allowing $z$ to approach zero, we have

$$
\begin{aligned}
\vartheta_{1}{ }^{\prime}(0) & =2 q^{1 / 4} \prod_{v=1}^{\infty}\left(1-q^{2 v}\right)^{3} \\
& =2 q^{1 / 4} \prod_{v=1}^{\infty}\left(1-q^{2 v}\right)\left(1+q^{v}\right)^{2}\left(1-q^{v}\right)^{2} \\
& =2 q^{1 / 4} \prod_{v=1}^{\infty}\left(1-q^{2 v}\right)\left(1+q^{2 v}\right)^{2}\left(1+q^{2 v-1}\right)^{2}\left(1-q^{2 v}\right)^{2}\left(1-q^{2 v-1}\right)^{2} \\
& =2 q^{1 / 4} \prod_{v=1}^{\infty}\left(1+q^{2 v}\right)^{2}\left(1-q^{2 v}\right) \cdot \prod_{v=1}^{\infty}\left(1+q^{2 v-1}\right)^{2}\left(1-q^{2 v}\right) \\
& \cdot \prod_{v=1}^{\infty}\left(1-q^{2 v-1}\right)^{2}\left(1-q^{2 v}\right) \\
& ={ }_{2}(0){ }_{3}(0) \vartheta_{4}(0),
\end{aligned}
$$

the last member being obtained by comparison with (11), (12), and (13).

UNIVERSITY OF ILLINOIS 